\title{
NOTICIARIO
}

\section{ARQUEOLOGÍA DEL PAISAJE: DE LA REFLEXIÓN A LA PLANIFICACIÓN ${ }^{1}$}

POR

\author{
ALMUDENA OREJAS \\ Universidad Alfonso X. Facultad de Historia del Arte y Patrimonio
}

\section{RESUMEN}

La reciente aparición de un buen número de proyectos y publicaciones sobre territorios y paisajes antiguos constituye un motivo de reflexión sobre los derroteros que está tomando la construcción de esta linea de investigación. No solamente es urgente evaluar el desarrollo teórico y metodológico y el papel jugado por las técnicas aplicadas, sino también analizar sus conexiones con el tan debatido mundo de la «arqueologia de gestión" y con las revisiones del concepto de patrimonio

\section{SUMMARY}

The recent apparition of a good number of projects and publications about ancient territories and landcapes constitutes a motiv of thougt on the direction this line of reseach is taking. The is not only a need to evaluate the theoretical and methodological development, the place that applied tecniques are playing, but also to analise its conexion with the so much debated "survey archaelogy" and the new approaches to the concept of historical heritage.

En los últimos años los denominados genéricamente "estudios territoriales» han experimentado un notable auge que se manifiesta en un incremento del número de trabajos y en una mejor calidad de los mismos, pero también ha dado lugar a la adopción de esta etiqueta en una serie de investigaciones que poco tienen en el fondo de novedosas. Sin duda el análisis territorial en el mundo antiguo y la

' Estas páginas han sido redactadas a partir de dos trabajos anteriores: una intervención en el Congreso Internacional $\mathrm{La}$ Peninsula Ibérica en la Antigüedad: la imagen de un territorio (Toledo, mayo de 1993), con el título "Territorio, análisis territorial y arqueología del paisaje" y la participación en un ciclo de conferencias sobre El paisaje rural en la Antigüedad, organizado por el Depto. de Historia Antigua de la Univesidad de Sevilla en mayo 1995 («Arqueologia del paisaje: balances y perspectivas")).
Arqueologia del paisaje exigen una reflexión conjunta de historiadores y arqueólogos, que nos haga tomar conciencia de los aspectos conflictivos, debilidades, dudas y perspectivas de trabajo, superando falsas oposiciones - conceptuales, metodológicas, etc.- y compartimentaciones cronológicas casi siempre ficticias. El mundo teórico desarrollado en este campo - sobre todo el anglosajón - resulta farragoso, con frecuencia cargado de una retórica que provoca «la huida" (Hernando 1992, 12), sin embargo, no cabe duda de la necesidad de conceptualización. Hay aún otro aspecto que necesariamente tenemos que tener en cuenta: es imprescindible la elaboración de proyectos de investigación coherentes con estas reflexiones, con los planteamientos teóricos y metodológicos y que esta coherencia se extienda al mundo de la gestión y conservación.

Los puntos de vista expuestos aqui han nacido de los trabajos llevados a cabo en el Departamento de Historia Antigua y Arqueologia del CSIC en la línea de investigación sobre Estructura social y territorio en la que se inscriben varios proyectos, entre ellos el de la Zona Arqueológica de las Médulas dentro del que ha surgido una reflexión en estos ámbitos, siempre en la perspectiva del análisis histórico. Dentro de esta necesidad de pensar la $\mathrm{Ar}$ queologia del paisaje resultaría pretencioso tratar de sistematizar de una forma global el tema ya que las formaciones y trayectorias actuales de los investigadores son muy diversas y el panorama en que nos movemos se resiste a una clasifícación simplista, oscilando entre la riqueza de planteamientos y la amenaza de la dispersión absoluta.

En primer lugar, y aunque parezca una cuestión obvia, creo que es necesario considerar por qué los estudios territoriales que conciernen al mundo anti- 
guo atraen cada vez a más investigadores y aglutinan un número creciente de perspectivas. Esto supone pensar en nuestro origen reciente, haciendo referencia a dos aspectos en absoluto disociados. Por una parte a lo que podriamos denominar el contexto científico, es decir la evolución de la Historia Antigua y de la Arqueología en los últimos años, desde las aportaciones de la Nueva Arqueología o la ecologia histórica a las revisiones radicales más recientes. En el caso concreto de la Arqueología, a lo largo de la década de los ochenta la Arqueología del paisaje ha ido suplantando en nuestros estudios a la Arqueología espacial y agrupando los análisis territoriales sobre el mundo antiguo; este cambio terminológico - pese a que en ocasiones no pase de ser una simple cuestión de estética o moda- responde a una nueva realidad científica y social desde que, en los primeros años de la década pasada, se sentaron las bases de la superación tanto de los enfoques fenomenológicos como de los derivados de la Arqueologia procesual. En España durante algunos años la confusión general fue grande ya que, en el marco de una tradición más ligada al regionalismo, irrumpieron simultáneamente las propuestas de la Nueva Arqueología y las críticas que empezaron a dar forma al postprocesualismo, en un ambiente marcado en cualquier caso por las corrientes anglosajonas ${ }^{2}$.

Evidentemente nada de esto es ajeno al contexto social. Baste con mencionar un aspecto sobradamente significativo: a lo largo de la segunda mitad del siglo XX asistimos a una reivindicación del espacio y de determinados recursos como bienes escasos y caros. Relacionados con este ambiente se detectan un buen número de movimientos y posturas, $y$ en él reside una buena parte del éxito de las propuestas ecologistas (en sus múltiples vertientes y «calidades»). Del mismo modo podemos reconocer una especie de bucolismo hacia el mundo rural y sus formas de vida y trabajo tradicionales (estrechamente ligado a su extinción), con un fuerte poder de evocación, generando lo que se ha denominado como "eco-antropología». Dentro de este contexto adquiere un papel singular la nueva concepción de patrimonio cultural que rompe con la tradicional idea de «lo antiguo» ligado a "lo monumental» $\mathrm{y}$ adquiere progresivamente una significación

\footnotetext{
${ }^{2}$ No hay que insistir en que desligar la Historia Antigua y la Arqueologia del resto del mundo cientifico es empobrecedor. Baste recordar que algunas de las tendencias que rápidamente he citado están estrechamente ligadas al auge de la Ecología o a la evolución de la Geografia y a la irrupción de la planificación del espacio como una necesidad primaria.
}

más dilatada (Darvill y otros 1993, 563-74), incorporando aspectos como la tecnología, la explotación de recursos y los mecanismos puestos en marcha para ello, la simbolización del espacio, etc. Es decir, subrayando en la idea de patrimonio no la yuxtaposición de elementos, sino la articulación que entre ellos existe, como reflejo de la dinámica social que dio lugar a su construcción y uso.

El cambio global que ha tenido lugar queda plasmado de una forma significativa en la terminologia empleada: del vocablo espacio (marcado por su carácter neutro o concebido como espacio físico marco de la actividad humana) se ha pasado al uso generalizado de los términos paisaje y territorio, el primero de ellos destacando la integración de «lo natural» $y$ «lo cultural» $y$ el segundo con un sentido eminentemente jurídico-político. En ambos casos queda claro que estamos ante categorías culturales.

En los ámbitos teórico y metodológico nos enfrentamos a una serie de cuestiones de base, causantes en buena medida de la dispersión a la que nos referíamos al principio y de las desiguales tendencias bajo titulos muy parecidos. Ante todo tenemos que afirmar que no se ha elaborado una teoría propia, ya que hasta hace pocos años no ha existido en este campo una reflexión efectuada por historiadores y arqueólogos. Progresivamente se han ido incorporando con desigual éxito enfoques, teorias, conceptos y métodos de otros ámbitos, en especial de la Geografia ${ }^{3}$ y de la Antropología, dando más una impresión de acumulación de ideas que de auténtica asimilación. A esto hay que añadir una escasa receptividad por parte de los investigadores dedicados al mundo antiguo. Todo ello genera una falta de definición que ha sido la principal causa del carácter restrictivo de una buena parte de los trabajos: la limitación de los estudios a análisis morfológicos descriptivos o a la inclusión de apéndices dedicados a exponer datos medioambientales que poco tienen que ver con el resto de la investigación.

Nuestro objeto de estudio puede resultar escurridizo y llevar rápidamente al escepticismo si no empezamos por hacer explícitos algunos puntos de partida a veces no tan evidentes. Si se toma como punto de partida a) que, en sentido estricto, nunca llegaremos a reconstruir el paisaje antiguo, b) que los actualismos nos impiden acceder a las raciona-

\footnotetext{
${ }^{3}$ M. de Bolós (dir.), Manual de Ciencia del Paisaje. Teorias, métodos y aplicaciones, Barcelona, 1992.
} 
lidades espaciales del pasado (plasmadas en determinadas decisiones locacionales) y c) que el paisaje se reduce a su superficie visible, entonces el paisaje será un objeto de estudio inasible. Por el contrario, para obtener una auténtica conceptualización del paisaje como objeto histórico, podemos asumir que:

1. El uso del espacio - en sentido amplioestá estrecha y directamente relacionado con su morfología (afirmación que no implica asumir relaciones automáticas entre formas y funciones) y esta morfología puede ser estudiada con metodología arqueológica.

2. De aquí podemos pasar a estudiar la sociedad, cómo y por qué actuaron así las comunidades que pensaron, materializaron y usaron ese espacio: su nivel de complejidad, su imbricación en instancias superiores, su grado de autonomía, su comportamiento en diversos ámbitos. Es decir, expresado a la inversa, se trata acceder al análisis de la sociedad a través del conocimiento de sus conductas (racionalidades) espaciales, integradas en una racionalidad (aunque sea una racionalidad limitada) y en unas pautas globales de conducta plasmadas en la morfología de ese espacio.

A partir de este momento dejamos de hablar de espacio (como término neutro o como objeto de contemplación), y nos estamos refiriendo ya a paisaje y territorio, de forma que podemos - y esta es una de nuestras tareas - fijar las bases metodológicas para la investigación: definición de categorías, parámetros, etc. Ese paisaje y ese territorio tienen ante todo un carácter sintético: es el espacio usado, diseñado, pensado, apropiado, sacralizado, abandonado... no es tan sólo un reflejo plano y estático de las comunidades. La sociedad conforma ese espacio generando paisajes pero a su vez el paisaje se convierte en un elemento activo en esa sociedad. Los conceptos y la terminología empleados en algunos trabajos recientes subrayan esta visión compleja y la necesidad de integración:

- El título de un artículo publicado hace unos meses por J. McGlade (McGlade 1995) es «Ecodinámica de los paisajes modificados por el hombre». En estas páginas el autor llega a afirmar que no hay medioambiente, no hay ecosistemas, sólo sistemas socio-naturales.

- La definición de categorías que subrayan aspectos perceptivos, simbólicos: es el caso de la categoria «visibilidad» propuesta por F. Criado (Criado 1993).

- La idea de «ritmos», causantes de aparentes contradicciones, como alternativa a los marcos cronológicos fijos y estandarizados (Orejas 1993 en prensa). La idea de evolución se distancia de las visiones lineales y se plaga de discontinuidades, rupturas, inestabilidades.

Para acceder al estudio del paisaje contamos con diversas fuentes y herramientas que en cualquier caso han de considerarse de forma integrada. La metodología ha de garantizar el paso de los datos y documentos (el registro arqueológico, las fuentes) al análisis del paisaje como síntesis social, mediante la definición de categorías y parámetros y de relaciones entre ellos que permitan el análisis morfológico «dirigido». Hay diversos tanteos y algunas largas experiencias: en el análisis de morfología agraria contamos con la trayectoria desarrollada en el Centre de Recherches d'Histoire Ancienne de Besançon. Otros casos, por citar dos ejemplos de la Prehistoria/ Protohistoria española son la definición de la ya citada categoría «visibilidad» en los trabajos coordinados por Felipe Criado (Criado 1991 y 1993), o la definición de factores y componentes tomada de la Geografia agraria propuesta por J. Vicent (Vicent 1991, 31-117). En el caso del proyecto Zona Arqueológica de Las Médulas hemos optado por la definición de una serie de parámetros (cualitativos y cuantitativos) que combinados nos permiten efectuar un análisis morfológico de detalle de ocupación y explotación del área estudiada (Fernández-Posse - Sánchez-Pelencia 1988, 83-228; Orejas 1993 en prensa).

El diseño de una metodología específica está ligado a la forma de acercamiento al paisaje. No entraremos ahora en la abundante literatura generada por la teoría del espacio (Bermejo 1992; Sanz 1993), tema que desborda con mucho nuestro campo de trabajo, sin embargo puede resultar esclarecedor resumir en cuatro grandes apartados las diversas aproximaciones al tema:

a) Las que simplemente consideran el paisaje como algo dado, en cuyo análisis, por lo tanto, no es necesario entrar. La superación de la oposición entre lo natural y lo cultural da al traste con este tipo de visiones. Esta propuesta es la asumida tradicionalmente en los estudios que daban por zanjado el tema con una «introducción geográfica» al estilo regionalista o con un capítulo dedicado al «medio fisico"n.

b) Las apoyadas en el funcionalismo y en los enfoques ecológicos, desarrolladas a partir de la de finición de cultura como una "forma de adaptación extrasomática al entorno", de forma que la complejidad social se mide directamente a través del grado de diferenciación funcional en el seno de una comunidad. Aquí encontramos las propuestas surgidas desde la revolución que supuso la Nueva 
Arqueologia, en especial la Arqueología Espacial (apoyada en el análisis locacional) y el análisis de captación económica. Por encima de las múltiples criticas y revisiones no podemos negar que su herencia es importante, ya que a ellas debemos la aceptación en el análisis histórico de la dimensión espacial y de las relaciones sociedad/ entorno en términos económicos, asi como el desarrollo de un importante volumen de herramientas y de técnicas de trabajo (modelos espaciales, técnicas de cuantificación). Pero también estas tendencias dieron forma y difundieron la idea de un "espacio científico y moderno" reductible a modelos, cuantificable, básicamente económico y regido por lazos funcionales ${ }^{4}$. Esta idea ha sido centro de muchas de las críticas realizadas por enfoques posteriores.

c) Un acercamiento que parte básicamente de una noción de paisaje como expresión de la nación, muy bien representada desde el siglo pasado en las arqueologias nacionales y en algunos trabajos localistas que pretenden descubrir plasmada en el paisaje una conciencia nacional, racial, étnica. En este enfoque el paisaje está caracterizado por su atemporalidad y la supervaloración de las permanencias que materializan ese espiritu.

d) Las posturas más recientes resultan menos fáciles de sistematizar: en general tienen en común la consideración del paisaje como resultado de la actividad humana, subrayando los aspectos relacionales e interpretativos frente a los formales ${ }^{5}$.

A excepción de la primera postura $-\mathrm{y}$ en algunas ocasiones la tercera-, en los enfoques citados tenemos que reconocer una intención común: la superación de un descriptivismo complejo apoyado en una yuxtaposición de datos de diverso origen, es decir, la superación de la Arqueología objetual.

Uno de los campos que mayor desarrollo ha experimentado en los últimos años es el estudio de la morfología de los paisajes antiguos. Dentro de ella los ámbitos de desarrollo tradicionales son el espacio agrario, que hizo entrar en la historia a comunidades campesinas, a lo no monumental, y las ciuda-

\footnotetext{
${ }^{4}$ P. J. Watson -S. A. LeBlanc- Ch. L. Redman, El método cientifico en Arqueologia, Madrid, 1974, pp. 116-119 y 135-138.

${ }^{3}$ En la investigación española contamos con una interesante serie de investigaciones: los trabajos ya citados de J.M. Vicent, en especial el publicado en 1991 (paisaje agrario como sintesis de relaciones sociales, analizadas mediante un enfoque arqueogeográfico) y de F. Criado (desde una visión que el autor califica como "culturalista") o los de F. Nocete en los que el paisaje se considera como base y efecto de una sociedad, entendiendo que "la territorialidad que adquiere uns sociedad no es nunca su reflejo, sino su base y efecton (Nocete 1988).
}

des, junto al estudio de la red de comunicaciones. Entre otras cosas, la Arqueologia agraria ha tenido que luchar, en los últimos veinticinco años, por soltar un lastre arrastrado desde la década de los treinta: la consideración de que el paisaje - entendido como paisaje agrario - era una creación medieval. A su avance han contribuido tanto los estudios sobre restos de estructuras agrarias fosilizados o reutilizados como sobre el poblamiento, así como el análisis de potenciales de explotación y captación cuando no es posible detectar formas antiguas. Sólo más recientemente se van incorporando sectores más desatendidos, como es el caso de las explotaciones mineras, el papel de la captación de recursos o la integración de las formas de comercio. Aquí han entrado en juego no sólo las mejoras indudables en las herramientas y metodología de los arqueólogos e historiadores, sino también las aportaciones de los estudios paleoambientales: la paleoecología y la arqueologia medioambiental han permitido el acceso a técnicas analíticas - análisis de restos vegetales y animales, paleosuelos, etc. - y a proyectos diacrónicos (Guilaine 1991; Harris - Thomas, 1991; Balaam - Rackham, 1992; Beck - Delort, 1993; Audouze - Fiches 1993).

Sin embargo, estos avances nos hacen correr el riesgo de entrar en una inflación de documentación, de catálogos e inventarios de unos «objetos» hasta ahora ignorados por la Arqueología y la Historia, pero que, si no se integran en una estrategia global de investigación, no pasarán de ser objetos y sólo habremos conseguido un cambio de escala. El análisis morfológico procede habitualmente por jerarquización de formas: desde $\mathrm{M}$. Bloch el paisaje agrario es estudiado desde su unidad minima, la parcela. Al margen de pretender considerar el paisaje como unas muñecas rusas en el que cada forma queda incorporada a una forma superior, lo interesante es reconocer que la forma de relacionarse los distintos elementos (sea o no una jerarquía) nos hace entrar de lleno en el estudio de la articulación de diferentes niveles: la organización propia de la comunidad, la aparición de planificaciones, el grado de autonomía... Estas relaciones no pueden abordarse dentro de un análisis lineal, sino reconociendo la existencia de ritmos diversos, eliminando la idea de tiempos y espacios absolutos incapaces de explicar muchas de las contradicciones con las que el historiador y el arqueólogo se encuentran con tanta frecuencia ${ }^{6}$. Del mismo modo el análisis de las formas per se, sin tener en cuenta su génesis, se aleja muy poco de las posturas que consideran el paisaje como algo dado. El paisaje, como ya hemos dicho, no es yuxtaposición, pero tampoco su evolu- 
ción ha de leerse en términos de superposiciones, sino como dinámica histórica, como sintesis.

La incorporación de enfoques sobre la percepción y el comportamiento, enunciados hace más de dos décadas en las ciencias sociales (conductismo, etc.), han permitido descubrir una nueva perspectiva: el hombre es un ser que actúa con una racionalidad limitada, interferida por procesos cognitivos (que incluyen la percepción), y esta racionalidad es observable mediante su conducta, en el caso que nos ocupa la conducta espacial. Asi surge la reivindicación de la imagen (por ejemplo un «mapa mental») como nexo entre lo real y la conducta del individuo o grupo y que a su vez actúa modelando esa realidad y pasa a formar parte de ella, interfiriendo en las planificaciones (Plácido 1993 en prensa)?

En la crítica global al funcionalismo, al procesualismo en Arqueología se han incorporado estos conceptos y vías de análisis. A partir de ellos se han planteado algunas cuestiones que no podemos perder de vista, por ejemplo la necesidad de incidir en que los paisajes antiguos son el resultado de racionalidades antiguas y por lo tanto estamos cometiendo un fallo de base si los analizamos desde nuestra racionalidad (de sociedad capitalista, con un concepto de espacio-consumo, de rentabilidad, etc). Lógicamente esto tiene sus implicaciones en el nivel metodológico, a la hora de definir parámetros y categorías, y al adoptar un determinado concepto de espacio. Es en esta línea en la que se plantea la necesidad de recuperar junto a la explicación (que en los sesenta caracterizaba a la Arqueología «realmente científica») la comprensión. La comprensión supone la incorporación del paisaje imaginario, y las relaciones entre el paisaje material y el paisaje mental (lo construido y lo vivido como se ha enunciado en muchas ocasiones): el espacio sólo adquiere

\footnotetext{
' En esta linea hay que considerar los trabajos centrados en el estudio de las relaciones centro-periferia establecidas entre el mundo bárbaro y el Mediterráneo (T. C. Champion Centre \& periphery: comparative studies in Archaeology, Londres, 1989; B. Cunliffe, Greeks, Romans \& Barbarians. Spheres of interaction, Londres, 1988; M. Rowlands . J. M. Larsen K. Kristiansen (eds.), Centre and periphery in the Ancient World, Cambridge, 1987. En las investigaciones sobre el período imperial romano es necesario avanzar en este sentido al analizar las relaciones con el mundo provincial: las soluciones adoptadas sobre el terreno, los espacios regulados o planificados, el impacto sobre las anteriores situaciones, las formas de apropiación y su gestión, con las contradicciones que generan las nuevas relaciones políticas, sociales y económicas.

${ }^{7}$ D. Ley, Geography without Man. A Humanistic Critique, Oxford, 1980; Tuan, Y. F., Topophilia. A Study of Environment Perception. Attitudes and Values, Nueva York, 1974: Tuan, Y.F., Space and place. The perspectives of experience, Londres, 1979.
}

sentido cuando va acompañado de una lectura del individuo o de la comunidad, que genera determinados comportamientos, actitudes y formas de percepción, que a su vez influyen en la modelación fisica del espacio.

¿Qué implicaciones tiene todo esto a la hora de proponer un proyecto de trabajo concreto? Fijar la bisagra que articule las reflexiones que aqui hemos propuesto - o muchas otras posibles - con las experiencias reales no es tarea fácil, pero es imprescindible abordarla, es una de las labores urgentes pendientes en la construcción de una teoría y una metodología especificas del paisaje como un auténtico objeto de estudio histórico.

Entrar de lleno en los estudios de paisaje y territorio exige tanto superar el análisis de los mapas de puntos (me refiero a la ubicación del hábitat antiguo fundamentalmente) como de sus relaciones planas (limitadas a las vías de comunicación por ejemplo), $y$ hacer entrar en el juego relaciones espaciales tridimensionales y temporales, de forma que no quedemos bloqueados en una visión estática y podamos acceder a las articulaciones de las diversas formas, su origen y evolución. En este sentido hay que entender el interés por el tema de las fronteras $^{8}$, o por el estudio de las relaciones centro-periferia que citábamos más arriba.

Ya nos hemos referido en otro momento a los análisis morfológicos y precisamente en este campo hay una serie de aspectos a los que hay que prestar atención. Si admitimos - como ya hemos apuntado-que el paisaje no es únicamente su superficie visible sino que es resultado de una racionalidad, a partir de un análisis morfológico del paisaje (realizado con metodología histórica y arqueológica) podemos acceder al estudio de esa racionalidad, de prácticas y relaciones sociales, del comportamiento de las comunidades que estamos estudiando y su percepción de la realidad (incluyendo la forma en que esta percepción actúa sobre lo material). Es decir, detrás de una morfología agraria determinada, correctamente analizada y contextualizada, se pueden leer prácticas agrarias, evolución de fuerzas productivas, grado de desarrollo, formas de tenencia y propiedad, usos del suelo, jerarquización, etc.

Indudablemente esto está ligado a la superación de las áreas geográficas reducidas como

${ }^{8}$ Los dos últimos volúmenes (13 y 14) de Arqueología Espacial están centrados en el estudio de las fronteras (Teruel, 1989 y 1993 respectivamente). Ver también A. Ruiz M. Molinos 1993. 258-282. 
marco único de la investigación, y a la adopción de perspectivas globales. En relación con esto hay que considerar la puesta en entredicho de los niveles micro, semi-micro y macro surgidos de la Arqueología Espacial - acusados de favorecer visiones estáticas por la falta de articulación entre ellos- y de los limites y fronteras considerados como datos existentes a priori destinados a encuadrar una investigación, y no como una cuestión abierta. En este mismo sentido, ampliar el espacio físico del estudio no implica automáticamente estar haciendo estudios territoriales ni arqueología del paisaje: si las hipótesis de trabajo y la metodologia no han cambiado en absoluto lo único que hemos logrado es un cambio de escala.

Un peligro inmediato lo constituyen los automatismos: la relación directa entre formas y funciones que evita abordar un verdadero análisis. Algunos rasgos morfológicos se asocian tradicionalmente a determinadas funciones, como el caso de considerar que la existencia de elementos de delimitación destacados en un asentamiento implica necesariamente un carácter defensivo del mismo, sin tener presente, por ejemplo, la posibilidad de interpretarlos como rasgos externos de la cohesión del grupo. Por otro lado, es relativamente frecuente constatar que un aspecto morfológico por sí mismo no tiene sentido, y que puede pervivir durante un prolongado periodo de tiempo por inercia, con un carácter residual o reutilizado para una nueva función. $\mathrm{Al}$ asumir directamente estos nexos - a veces avalados por su reiteración en las publicaciones - bloqueamos cualquier posibilidad de hacer un auténtico análisis de las formas.

Otro de los temas insistentemente planteados a raiz de la expansión de los estudios territoriales es la relación entre el tiempo y el espacio, con algunas posturas extremas que llegan a la negligencia de uno $u$ otro (la consideración de que todo lo que no está articulado fundamentalmente en el tiempo es ahistórico, o propuestas en la línea de realizar lecturas exclusivamente espaciales). Habitualmente estos temas no están a nuestro alcance, ya que nuestra formación en este campo es francamente pobre, por ello, y salvo honrosas excepciones, resulta pretencioso y arriesgado ir más allá de algunas reflexiones globales que sirvan para arropar la visión adoptada en una investigación. En cualquier caso debemos huir del falso debate que opone tiempo y espacio: no son reductibles el uno al otro, y para un historiador es tan imposible un análisis aespacial como a-temporal. Ni espacial ni temporalmente son operativas las visiones lineales, por ello entramos en el problema de la crisis de uno de los apoyos más tradicionales del arqueólogo: la cronologia. Este debate tiene muchas implicaciones concretas en el análisis de la permanencia y la movilidad de los rasgos del paisaje o de las visiones estratigráficas, en las que lo vertical como lectura del tiempo y lo horizontal como lectura del espacio debe de ser superado.

Un aspecto más que todos nos planteamos de una forma más o menos explicita en alguna ocasión ( $y$ no sólo en los estudios territoriales y de paisaje) es el peligro de cometer anacronismos y actualismos, que se convierte en una cuestión metodológica cuando la selección de determinadas categorias y parámetros puede subrayarlos. Esto ocurre con frecuencia en la aplicación de conceptos como el de rentabilidad, o al reflejar en la investigación las valoraciones actuales, por ejemplo de la propiedad. En este sentido hay que mencionar trabajos como el ya citado de Juan Vicent (Vicent 1991), basado en una reinterpretación del site catchment analysis inspirada en: a) los planteamientos de Tchainov: el campesino precapitalista no tiende a maximizar el beneficio cuando el excedente es inútil en su sistema. Su racionalidad es hacer sólo el esfuerzo necesario, y b) en un modelo factorial del paisaje a nivel regional, en el que intervienen elementos (tiempos...) y factores (suelos, clases agrológicas...).

La diacronía es consustancial a este tipo de investigaciones. El paisaje es diacrónico, en el de hoy vemos el de siglos. Sin embargo, una auténtica lectura diacrónica es muy difícil y exige el concurso de informaciones de diverso origen. Algunas aparentes contradicciones entre documentación literaria, epigráfica y arqueológica desaparecen al considerarlas desde este ángulo de la diacronia (Ruiz-Molinos 1993. 258-82). De nuevo estamos ante un problema surgido de la falta de un desarrollo metodológico: la cuestión está en evitar hacer del origen de la información un criterio de jerarquización a la hora de evaluar su credibilidad, basado en que lo más explícito es más fiable. Desde este punto de vista se daria siempre prioridad a lo escrito $y$, a la hora de estudiar el registro arqueológico, a los datos de excavación sobre los de prospección.

Por otro lado, los documentos y técnicas de trabajo recientemente incorporados (teledetección, tratamiento informático de datos e imágenes, etc.), nos hacen asomarnos a un mundo que con frecuencia da la sensación de ser inabarcable (entre otras cosas por su rápida evolución) y, sobre todo, nos hace correr el riesgo de convertirnos en técnicos especializados, perdiendo la perspectiva general. Esto nos obliga a hacer un esfuerzo por reinstrumentalizar esos úti- 
les de trabajo, por otra parte garantes de una buena parte de los avances. El acceso a más información y a un tratamiento de los datos más adecuado puede ser fuente de confusiones si no no se logran insertar en un marco teórico y metodológico. Dando un paso más, sólo si los datos tienen sentido la proyección patrimonial será coherente.

Volviendo a la idea inicial de que nuestro auténtico centro de interés es el estudio de los procesos sociales, es fácil asumir que las barreras temporales no tienen sentido, $y$ al no hablar de un medio físico en abstracto sino de un paisaje, estamos asumiendo la posibilidad de actuar desde el presente, de intervenir. Aquí estamos en esa bisagra que articula las llamadas arqueología de investigación y arqueología de gestión (Ruiz Zapatero 1991, 7; Hernando 1992, 12 y 31; Casal 1995) y que permite abrir la puerta de las planificaciones, la previsión de riesgos, etc. Pero es también en este punto en el que el paisaje de los arqueólogos entra en conflicto con el de los constructores, gestores, ingenieros, geólogos... La clave de estas oposiciones está en la falta de claridad en las relaciones entre los diferentes niveles de la investigación arqueológica y su repercusión en las intervenciones patrimoniales.

Las grandes obras de infraestructura, el abandono y empobrecimiento de algunas regiones tradicionalmente agrarias, los procesos de desertización... están obligando a la realización de estudios de impacto, de planificación del territorio en términos de "desarrollo sostenible» ${ }^{9}$. Es evidente que cualquier espacio tiene restos arqueológicos, más o menos evidentes, que han contribuido y contribuyen a su evolución y configuración presente: evolucionan pero hay una memoria fosilizada de una u otra forma. El tipo y grado de evidencia de los restos es muy variable, desde abundantes, llamativos y contextualizados a pobres e inconexos, no obstante en muy contadas ocasiones constituyen un conjunto global e integrado.

Por lo tanto, al hablar de paisajes arqueológicos estamos usando una expresión ambigua que se puede referir a:

a) En sentido amplio a cualquier paisaje. Quizás haya que sustituir en este caso la expresión: puesto que todos los paisajes son históricos/ arqueológi-

\footnotetext{
"La expresión "sustainable development" surgió en la Comisión Mundial para el Medio Ambiente y el Desarrollo de 1987 , $y$ se difundió en el texto conocido como Informe Brundtland (Riechmann 1995).
}

cos, habria que hablar mejor de análisis histórico de paisajes.

b) En sentido más restringido $-\mathrm{y}$ esto enlaza con las cuestiones patrimoniales, de conservación, etc...- nos referimos a paisajes con restos materiales del pasado, que deben cumplir una serie de requisitos.

La fijación de estas condiciones ha sido abordada ya en algunos proyectos, es el caso de las que proponen Darvill y su equipo (Darvill y otros 1993, 564) para la definición de relict cultural landscapes:

1. Un espacio limitado y fosilizado, teniendo en cuenta que una unidad topográfica determinada es dificil que coincida con un espacio social, funcional y cognitivo. La determinación de este espacio exige subrayar los elementos internos de articulación (naturales como los ríos, artificiales como las vias, recursos, etc...).

2. La existencia de una evidencia arqueológica - en el sentido de restos materiales - suficiente, pero no entendida como una yuxtaposición de monumentos, de hitos.

3. La necesidad de ligar diversidad y coherencia en estos espacios.

4. Tener en cuenta una serie de factores esenciales a la hora de definir estos paisajes culturales, entre otros la globalidad y articulación, la escala y la visión sincrónica / diacrónica.

Una de las primeras necesidades que hay que cubrir es contar con un marco global legal que sea la base de iniciativas y desarrollos de proyectos y sobre la que se pueda trabajar. La Ley del Patrimonio española propuso en el año 1985 una de las concepciones más modernas de ese momento: en ella no se considera ya el patrimonio como sinónimo de «lo antiguo», ni de lo «monumental»" ${ }^{10}$. En esta misma tendencia, la ley incluye también la definición de Zona Arqueológica: «es el lugar o paraje natural donde existen bienes muebles o inmuebles susceptibles de ser estudiados con metodo logía arqueológica, hayan sido o no extraídos y tanto si se encuentran en la superficie, en el sub-

\footnotetext{
${ }^{10}$ «... forman parte del Patrimonio Histórico Español los bienes muebles e inmuebles de carácter histórico susceptibles de ser estudiados con metodología arqueológica, hayan sido o no extraidos y tanto si se encuentran en la superficie o en el subsuelo, en el mar territorial o en la plata forma continental. Forman parte, asimismo, de este patrimonio, los elementos geológicos y paleontológicos relacionados con la historia del hombre y sus origenes y antecedentes" (Ley de Patrimonio Español 16/85, art. 40.1).
} 
suelo o bajo las aguas territoriales españolas» (art. 15.5). Diez años después los problemas que han surgiendo en situaciones concretas, las dificiles relaciones de esta ley con otras normativas sobre medioambiente, ordenación urbana, etc. y la evolución de la Arqueología exigen una revisión y matizaciones ${ }^{11}$.

El debate en torno a la concepción del patrimonio aparece reflejado también en la documentación redactada desde el Consejo de Europa y la UNES$\mathrm{CO}$. En el caso de la CEE el interés se centra (hasta ahora) casi exclusivamente en comercio y tráfico de obras de arte (Derout 1993) y las definiciones que se barajan tienen a veces un tono decimonónico: por ejemplo, la consideración de los bienes culturales protegidos como «tesoros nacionales, con valor artístico, histórico y arqueológicon, implica por un lado la excepcionalidad («tesoros») y por otro lado una dudosa adjudicación nacional.

Sin embargo está ya sobre la mesa el problema de la definición y gestión del "patrimonio común europeo", asi como la consideración de los paisajes como parte de ese patrimonio común ${ }^{12}$ : ¿es el patrimonio europeo igual a una selección de esos «tesoros nacionales»? ¿Cómo resolver los posibles conflictos entre legislaciones (patrimoniales, del suelo, medioambientales...) nacionales?. Hasta el momento los documentos más útiles son las recomendacio-

\footnotetext{
"Otra categoria contemplada (dentro de los bienes inmuebles) en la Ley del Patrimonio histórico español de 1985, dentro de esta misma intención es la de Sitio Histórico, definido como «el lugar o paraje natural vinculado a acontecimeintos o recuerdos del pasado, a tradiciones populares, creaciones culturales o de la Naturaleza y a obras del hombre, que posean valor histórico, etnológico, paleontológico o antropológicon. Tanto en el caso de las zonas arqueológicas como en el de los sitios históricos, la declaración de Bien de Interés Cultural (BIC) pasa por la elaboración de un Plan Especial de Proteción municipal (art. 20). Hay que observar que en estas figuras, al superar el nivel objetual, la disociación entre la naturaleza y la actividad humana se hace imposible. La relación entre estos párrafos y los textos sobre espacios naturales protegidos - en los que en buena medida se inspiran- es evidente (Ley 15/1974 de espacios naturales protegidos y R.D. $2676 / 1977$, Ley $4 / 1989$ de conservación de espacios naturales y de la flora y fauna silvestres, art. 16 y 17 entre otros).

En la Ley sobre régimen del suelo y ordenación urbana (1986), en el artículo 12 se indica que los Planes Generales Municipales de Ordenación deben procurar la "defensa del paisaje». El artículo 19 de dicha ley se centra en los elementos que hay que tener en cuenta al plantear «la protección del paisaje para conservar determinados lugares o perspectivas". Sin embargo en ella se siguen privilegiando las «bellezas naturales", lo pintoresco, lo singular.

12 Asi se plantea en la acción COST G2 PASTA, Paysages antiques et structures rurales: textes et archéologie, en la que se integran una decena de paises con varios equipos que trabajan en esta línea de inveatigación.
}

nes y propuestas de trabajo, que esbozan líneas más avanzadas, apoyando claramente una concepción más dilatada del patrimonio, que rompe con la limitación de la obra, del objeto, y que tiene una tendencia claramente integradora: confusión de lo naturalantrópico, usos tradicionales del suelo en sentido diacrónico ${ }^{13}$. Desde esta perspectiva, no es suficiente la elaboración de catálogos e inventarios, ya que la aproximación social, económica, cultural, no debe ser diferente a la patrimonial y no está en absoluto desligada de la investigación.

El borrador de la recomendación del CE sobre la conservación de paisajes culturales de 1994, subraya la necesidad de formar técnicos, profesionales en este ámbito, que sigan una formación específica, e incluso se propone un esbozo de plan de estudios. Se trata de profesionales capaces de ligar investigación, divulgación, conservación, gestión, de poner a punto procesos de identificación y evaluación, asi como los métodos y herramientas de intervención. Efectivamente, uno de nuestros problemas es el abismo entre la verborrea de la conceptualización, por un lado, la construcción de una metodología especifica, por otro, y los problemas de gestión: podemos escribir páginas y páginas $\mathrm{y}$, al llegar un proyecto a nuestras manos, hacer un análisis convencional, en un marco regionalista.

Es imprescindible tener presentes las «otras concepciones del paisaje»: el de los constructores, el de las administraciones... Abordar la conservación y gestión exige medir por un lado el valor arqueológico global y el grado de coherencia que existe entre los objetivos y otros intereses. Es decir, no tiene sentido un conservacionismo radical, ni una mitificación de los restos con el único argumento de su cronología. Estamos ante un objeto de estudio común a múltiples disciplinas, en el que confluyen intereses, puntos de vista, tradiciones académicas y de investigación y técnicas diferentes: esta realidad no puede ser ignorada por el historiador-arqueólogo que puede convertirse en sinónimo de reaccionario.

${ }^{13}$ Entre los documentos y actuaciones más recientes y centrados en el tema que nos ocupa podemos citar la recomendación de 1980 del comité de ministros del Consejo de Europa sobre la formación especializada de arquitectos, urbanistas, ingenieros y paisajistas, la campaña del Consejo de Europa en 1986-87 sobre el medio rural, la convención de Malta de 1992 sobre el patrimonio arqueológico, la directiva de la Comunidad Europea sobre la conservación de hábitats naturales y semi-naturales de 1992 y la recomendación de Consejo de Europa (1994) sobre la conservación de los paisajes culturales. A todo ellos hay que añadir los escritos sobre medioambiente, estrechamente relacionados con todo lo anterior. 
El patrimonio no sólo no es una rémora para el desarrollo, sino que puede convertirse en su protagonista, en un recurso de primer orden, en especial en zonas marginales y con escasos posibilidades de otro tipo ${ }^{14}$. Los proyectos de parques arqueológicos y culturales - superando la idea de yacimientos visitables o yacimientos-museos-, museos, ecomuseos, itinerarios son potenciales dinamizadores económicos, sociales y culturales de las zonas en que se instalan ${ }^{15}$. La puesta en marcha de estas actividades pasa por aclarar y desarrollar algunas cuestiones clave:

1. Dentro del mundo arqueológico: a) es necesario acabar con la tradicional actitud de «ensimismamiento" y abordar la proyección social de las investigaciones y b) romper la identificación arqueólogo-excavador, contradictoria con las tendencias más recientes y las concepciones patrimoniales actuales.

2. En otro terreno son necesarias legislaciones nítidas y no contradictorias, tanto entre los diferentes niveles de la administración, como entre las legislaciones patrimoniales, del suelo, regulación de espacios urbanos y de espacios naturales, etc. (Alonso 1992).

3. Revisión de las teorías y metodologías de conservación y no-destructivas (non-destructive survey).

4. La creación, la imaginación de nuevas for-

\footnotetext{
${ }^{14} \mathrm{La}$ relación del medio ambiente con el desarrollo económico ha suscitado hasta ahora un interés mucho mayor que las posibilidades del patrimonio histórico. No obtante, las siguientes palabras de Naredo son reveladoras en ambos terrenos: «En suma, que junto a los empeños de estudiar el «medio ambiente» desde la economia standard, se observa también un repensar la economia desde el «medio ambiente», que está por primera vez abriendo el viejo universo de lo económico, trasladando su centro de gravedad y relativizando sus categorias básicas. El desplazamiento sordo de las preocupaciones hacia aspectos patrimoniales, fisicos e institucionales exteriores al universo de la economia stan$d a r d$, ha inducido también a abrir la "caja de Pandora» del mercado, que se creía siempre portadora de soluciones "óptimas». Se vio entonces con sorpresa que tras la supuesta umano invisible" del mercado se encuentra la mano bien visible de las instituciones que determina sus resultados, desatando asi un proceso general de revisión y relativización de la capacidad explicativa de los viejos enfoques y categorias de los económico, recogido recientemente en una de las publicaciones internacionales más prestigiosas que informa sobre la evolución del pensamiento cientifico" (se refiere a: W. B. Arthur, «Pandora's marketplace», New Scientist, 6-2, 1993").

is Como proyecto sobre un yacimiento es interesante el trabajo realizado en Flag Fen por el equipo de F. Pryor (Pryor 1989). La figura del Parque Arqueológico - para el que se han propuesto definiciones muy diversas- ha generado en los últimos años una abundante literatura y ha sido protagonista de reuniones y seminarios: Seminario de Parques Culturales (Madrid, 1989), Madrid, 1993 (en especial las intervenciones de M. A. Querol y de C. Martín de Guzmán); A. García L. Caballero 1991; M. T. Costales 1995.
}

mas de comunicación e integración entre el pasado $y$ el presente.

Nos enfrentamos ya a una serie de problemas y preguntas específicas, sintoma evidente de emancipación, pero nunca de autosuficiencia, contraria al análisis de las dinámicas históricas y a la sintesis.

\section{BIBLIOGRAFÍA}

Alonso lbẫez, M. R.: El patrimonio histórico. Destino público y valor cultural, Madrid, 1992.

Analyse des paysages, Analyse des paysages: de la terre au ciel. Table-Ronde International de l'Uni versité de Besançon (Besançon, 1993). París, 1995.

Audouze, F., y Fiches, J. L.: «L'Archéologie française et les paléo-environnements", Annales ESC, 1993, 1, 1993, pp. 17-41.

BALAAM, N., y RaCkAM, J. (Eds.): Issues in Environmental Archaeology. Perspectives on its Public Role, Londres (Inst. of Archaeology, University College London), 1992.

BARKER, G., y LloYd, J. (Eds.): Roman Landscapes: Archaeological survey in the Mediterranean region, Londres (British School at Rome. Archaeological Monographs), 1991.

Beck, G., y Delort, R. (Eds.): Pour une histoire de l'environnement. Travaux du programme interdisciplinaire de recherche sur l'environnement, París, 1993.

Bermejo, J. C.: «Des dimensions significatives de l'espace historique», Dialogues d'Histoire Ancienne, 18, 2, 1992, pp. 29-49.

Bolos, M. de (dra.): Manual de ciencia del paisaje. Teorias, métodos y aplicaciones, Barcelona, 1992.

CASAl García, R.: "Arqueología y política», Actas del XXII Congreso Nacional de Arqueologia (Vigo 1993), vol. I, Vigo, 1995, pp. 239-240.

Chouquer, G.: «Que reste-t-il de 3000 ans de création paysagère?», Études rurales, 121-124, 1991, pp. 45-58.

Chouquer, G.: "Chronique. Parcellaires, cadastres et paysages», Revue Archéologique du Centre de France, 32, 1993, pp. 205-230.

Chouquer, G., y FAvory, F.: Les paysages de l'Antiquité. Terres et cadastres de l'Occident romain (IVe s. av. J.-C./ IIIe s. ap. J.-C.), París, 1991.

Costales, M. T.: «Los parques arqueológicos en Españan, Actas del XXII Congreso Nacional de Arqueologia (Vigo 1993), vol. I, Vigo, 1995, pp. 249-252. 
Criado BoAdo, F.: «La Arqueología entre el Tiempo y el Espacio: límites de la Arqueología del Paisaje", II World Archaeological Congress (Methodology and and Theory in Landscape Archaeo$\log y$ ). Barquisimeto, (Venezuela), 1990.

Criado Boado, F. (Dir.): Arqueología del Paisaje. El área Bocelo - Furelos entre los tiempos paleoliticos y medievales (Campañas de 1987, 1988 y 1989), Santiago, 1991.

CRIADO BOADO, F.: «Visibilidad e interpretación del registro arqueológico», Trabajos de Prehistoria, 50,1993 , pp. $39-56$

Darvill, T., Gerrard, C., y Startin, B.: «Identifying and protecting historic landscapes", Antiquity, 67, 1993, pp. 563-574.

Derout, A.: La protection des biens culturels en droit communautaire, Rennes, 1993.

Environmental Archaeology, Experimentation and Reconstruction in Environmental Archaeology. Symposia of the Association for Environmental Archaeology. Roskilde, Dinamarca, 9, Oxford, 1990.

Fernández-Posse, M. D., y Sánchez-Palencia. F. J.: La Corona y el Castro de Corporales II. Campaña de 1983 y prospecciones en la Valderia y la Cabrera (León), Madrid (EAE 153), 1988.

Garcia Blanco, A., y Caballero, L.: «La comunicación del Parque Arqueológico", Jornadas Internacionales sobre Arqueologia de Intervención, San Sebastián, 1991, pp. 375-409.

Greeves, T.: «Archaeology and the Green movement: a case for perestroika", Antiquity, 63, 1989, pp. 659-666.

Guilaine, J. (Ed.): Pour une Archéologie Agraire, Paris, 1991.

Harris, D. R., у Thомas, K. P. (Eds.): Modelling Ecological Change. Perspectives from Neoecology, Paleoecology and Environmental Archaeology, Londres (Inst. of Archaeology, Uni. College London), 1991.

Hernando, A.: "Enfoques teóricos en Arqueología», SPAL, 1, 1992, pp. 11-35.

Legislación, Legislación sobre patrimonio histórico (ed. de J. García Fernández), Madrid (Tecnos), 1987.

Leveau, PH., Silliéres, P., y H Campagnes de la Méditerranée romaine, Paris, 1993.

MCGLADE, J.: «Archaeology and the ecodynamics of human-modified landscapes», Antiquity, 69, 1995, pp. 113-132.

NAREDO, J. M.: «Repensar la economía desde el medio ambiente», De la economía a la ecologia, Madrid, 1995, pp. 37-42.
Nocete. F.: «Estómagos bipedos/ estómagos políticos», Arqueoloia Espacial, 12, Lisboa-Teruel, 1988, pp. 119-139.

Orejas, A.: «Arqueologia del paisaje. Historia, problemas y perspectivas", Archivo Español de Arqueologia. 64, 1991, pp. 191-230.

OREJAs, A.: Estructura social y territorio. El impacto romano en la Cuenca Noroccidental del Duero. Madrid, 1993 (en prensa).

Parques Arqueológicos, Seminario de Parques Arqueológicos (Madrid, 13-15 Diciembre 1989), Madrid (Ministerio de Cultura), 1993.

PAsta, Paysages et structures rurales: textes et archéologie, acción COST G2 1995-1998.

Plácido, D.: «La imagen simbólica de la Península Ibérica en la Antigüedad», La Peninsula Ibérica en la Antigüedad: la imagen de un territorio (Toledo, mayo 1993), en prensa.

PRYOR, F.: " 'Look that we've found'- a case study in public archaeology", Antiquity, 63, 1989, pp. 51-61.

Pryor, F.: «The reluctant Greening of Archaeology», Antiquity, 64, 1990, pp. 147-150.

RiechmanN, J.: «Desarrollo sostenible: la lucha por la interpretación», De la ecología a la economía, Madrid, 1995, pp. 11-35.

Ruiz, A., y Molınos, M.: Los iberos. Análisis arqueológico de un proceso histórico, Barcelona, 1993.

Ruiz Zapatero, G.: «Arqueología y Universidad. La reproducción del sisteman, Revista de Arqueologia, 1991 (febrero), pp. 6-7.

Sánchez-Palencia, J., Férnandez Posse, M. D., Fernández Manzano, J., Orejas, A., Álvarez, Y., y López, L.: Zona Arqueológica de Las Médulas: estudio de viabilidad para su conversión en Parque Arqueológico (informe presentado al Ministerio de Cultura), Madrid, 1992.

SAnZ, N.: «Para una lógica social del espacio en Prehistoria», Complutum, 4, 1993, pp. 239-252.

Sites culturels, Proposition de récommendation aux États membres relative à la conservation des sites culturels integrée aux politiques du paysage (rapport intérimaire d'activité du Groupe de Spécialistes sur les sites et paysages culturels, Estrasburgo (Comité du Patrimoine Culturel), 1994.

SMITH, L.: «Heritage management as postprocessual archaeology?», Antiquity, 68, 1994, pp. 300-309.

ViCENT, J. M.: «Fundamentos téórico-metodológicos para un programa de investigación arqueogeográfica», El cambio cultural del IV al II milenios a.C. en la comarca Noroeste de Murcia, (P. López, de.), Madrid, 1991, pp. 29-117. 